\title{
Development of a parallel local oxidation nanolithography instrument
}

\author{
Javier Martinez and Nuria S. Losilla \\ Instituto de Microelectrónica de Madrid, CSIC, Isaac Newton 8, Tres Cantos, Madrid 28760, Spain \\ Fabio Biscarini \\ CNR-Istituto per lo Studio dei Materiali Nanostrutturati (ISMN), Via P. Gobetti 101, I-40129 Bologna, Italy
}

Georg Schmidt, Tanja Borzenko, and Laurens W. Molenkamp

Physikalisches Institut, Universität Würzburg, Am Hubland, 97074 Würzburg, Germany

Ricardo Garcia ${ }^{a)}$

Instituto de Microelectrónica de Madrid, CSIC, Isaac Newton 8, Tres Cantos, Madrid 28760, Spain

(Received 27 February 2006; accepted 24 July 2006; published online 22 August 2006)

\begin{abstract}
We have developed an instrument to perform local oxidation nanofabrication processes in parallel. The instrument has three major components, the stamp holder, the sample base, and the supporting frame. The sample base is actuated by three precision screws that enable motion in the three orthogonal directions. Sample base and stamp holder are enclosed and sealed inside a chamber with two inlets to introduce different gases. The chamber is supported by a rigid frame. We show the parallel patterning of silicon oxide features on silicon surfaces by the application of a bias voltage between the sample and the stamp when they are in contact. Arrays of parallel lines separated by $100 \mathrm{~nm}$ have been patterned over $\mathrm{cm}^{2}$ regions in one minute. (C) 2006 American Institute of Physics. [DOI: 10.1063/1.2336773]
\end{abstract}

Local oxidation of semiconductor, metallic, and organic surfaces by atomic force microscopy (AFM) has established itself as a robust, reliable, and flexible lithographic method for the fabrication of nanometer-scale structures and devices. ${ }^{1-5}$ In scanning probe-based oxidation a water meniscus provides both the chemical species (oxyanions) and the spatial confinement for the anodic oxidation of a nanometersize region of the sample surface. ${ }^{6,7}$ The process is rather general because many different materials and/or surfaces have been patterned such as semiconductors, ${ }^{8,9}$ metals, ${ }^{10}$ dielectrics, ${ }^{11}$ perovskite oxides, ${ }^{1}$ or self-assembled monolayers. ${ }^{12}$ Furthermore, it has also been shown that the nanostructures could also be fabricated by using organic solvents such as ethyl alcohol, hexadecane, octene, and octane. ${ }^{6,13-15}$

This technique is very useful for prototyping a variety of nanoscale devices with designed functionality, however, it has limited technological implications due to the sequential character of the AFM technology. Recently, several authors have demonstrated that the local oxidation process could be upscaled in a straightforward way by using a solid support with multiple protrusions as the cathode electrode. ${ }^{16-20}$ Mühl et al. used a probe with a few protrusions to demonstrate that parallel oxidation was feasible. ${ }^{16}$ Hoeppener et al. used transmission electron microscope copper grids to replicate micrometer-scale patterns on a organosilane monolayer. ${ }^{17}$ Cavallini et al. transferred the bits of a metallized digital video disk (DVD) to a silicon surface ${ }^{18}$ and Yokoo has used silicon stamps modified by electron beam lithography to generate $300 \mathrm{~nm}$ pitch dot patterns. ${ }^{19}$

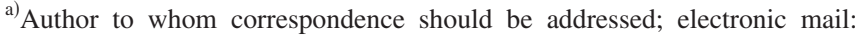
rgarcia@imm.cnm.csic.es
}

In general, the above instruments seem to lack of a mechanism to control both the pressure exerted on the sample and the alignment of the stamp on the sample. Furthermore, there are not detailed descriptions of the above instruments. Here, we report the development of a general purpose parallel patterning instrument to perform local oxidation nanolithography over macroscopic regions. The sample is approached towards the stamp with submicrometer accuracy. The sample base can pivot with respect to three fixed points. Submicrometer approach together with pivotal rotation allows to compensate an increase of the local pressure in a region of the sample. The performance of the instrument has been characterized by replicating arrays of periodic stripes with $100 \mathrm{~nm}$ lattice periodicities.

The parallel oxidation nanolithography system [Fig. 1(a)] has three main elements: (i) the stamp holder, (ii) the sample stage, and (iii) the supporting frame. The stamp is clamped laterally between two metallic wires, one is fixed while the other acts as a spring. This enables a user-friendly stamp exchange. The wires are attached onto a Plexiglass frame and connected to an external power supply. The sample base is a metallic piece with a small hole in the middle connected to a vacuum pump. The base has three degrees of freedom [Fig. 1(b)] which enable to distribute uniformly the pressure on the sample. The sample is placed on top of the base and it is fixed by the difference of pressure exerted by a vacuum pump. The frame sustains and encloses sample and stamp in a chamber with two inlets. Those inlets enable the introduction of different gas vapors such as water or ethanol. $3 \mu \mathrm{m}$ screws allow us to move the sample with respect to the stamp in the $x, y$, and $z$ directions. The approach and the lateral alignment are controlled optically by a microscope through a lateral window in the chamber. The fine $z$ approach is controlled electronically by detecting the 

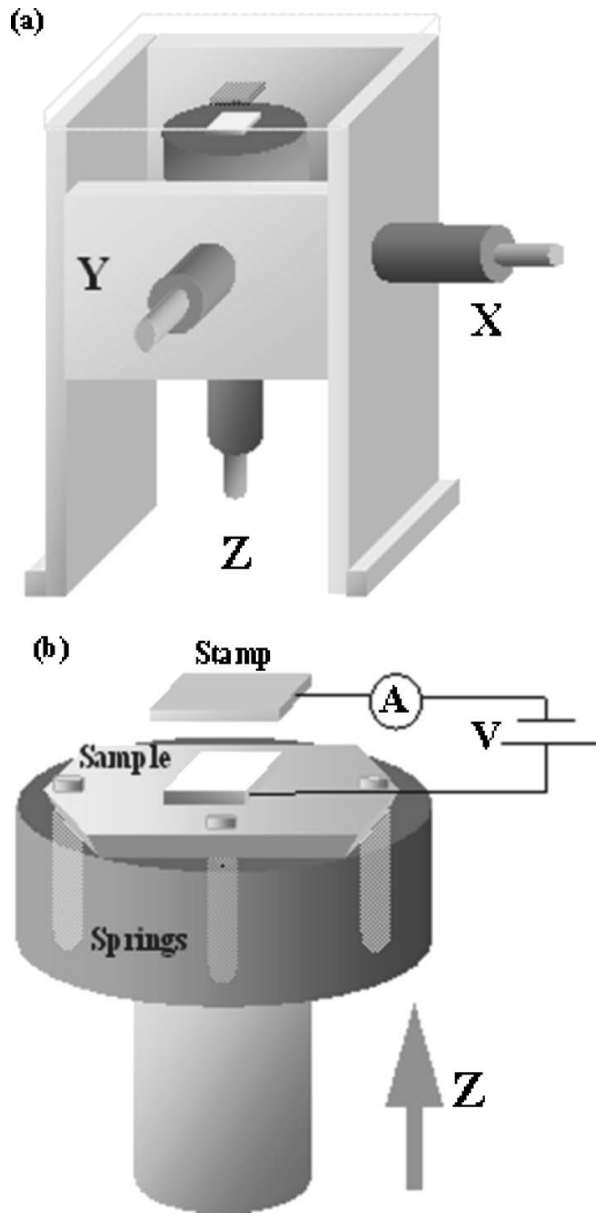

FIG. 1. Schematic description of the parallel oxidation instrument. (a) Overall view of the system. (b) Sample base, stamp holder, and electrical connections.

current flowing between the stamp and sample. A complete view of experimental system including accessories is presented in Fig. 2.

To test the performance of the parallel oxidation instrument we have prepared by e-beam lithography a silicon stamp with motives characterized by parallel stripes of $20 \mu \mathrm{m}$ in length, $200 \mathrm{~nm}$ in width, and $100 \mathrm{~nm}$ apart. The

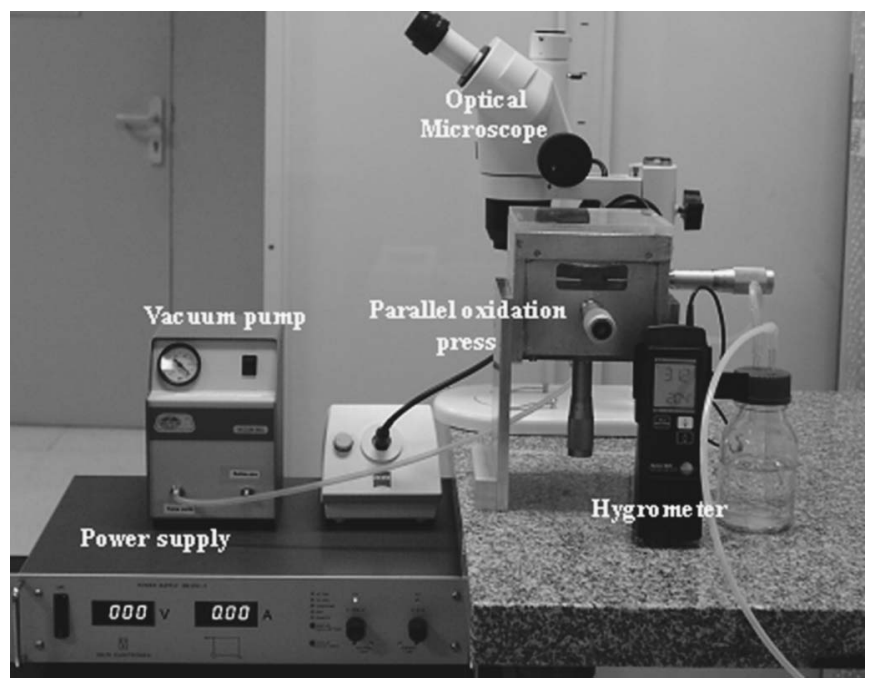

FIG. 2. Photograph of the complete experimental system.

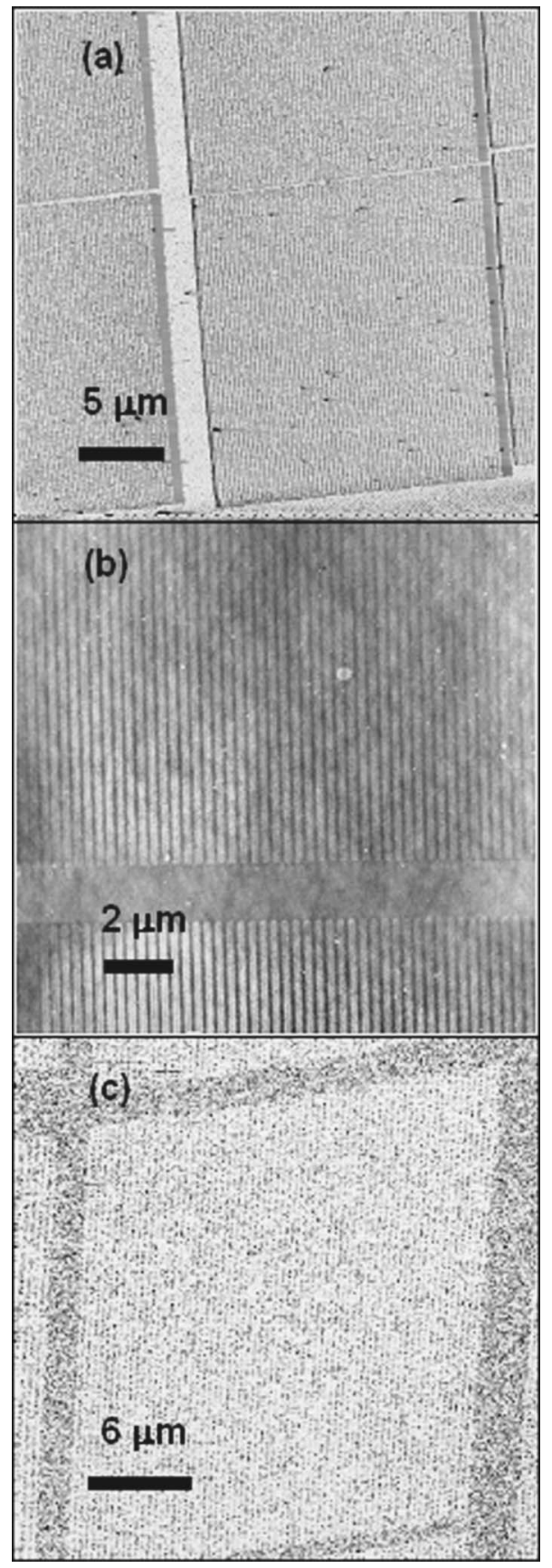

FIG. 3. (a) Amplitude modulation AFM image of a gold coated silicon stamp. The stamp consists of $20 \times 20 \mu \mathrm{m}^{2}$ rectangular patches. Each patch is an array of parallel lines with a $300 \mathrm{~nm}$ pitch. (b) AFM image of the parallel oxidation replica. Regions from two different patches are imaged. (c) AFM image of the parallel oxidation replica after etching in $\mathrm{KOH}$.

lines are grouped in $20 \times 20 \mu \mathrm{m}^{2}$ squares. Those squares form a mosaic that covers an area of $400 \times 400 \mu \mathrm{m}^{2}$. To improve the electrical conductivity, the stamp was coated with a $100 \mathrm{~nm}$ thick film of gold. Figure 3(a) shows an AFM image of representative region of the stamp. Figure 3(b) shows an AFM image of the parallel oxidation patterns formed after the application of a voltage pulse of $36 \mathrm{~V}$ for 1 min (relative humidity of $80 \%$ ). Etching in $\mathrm{KOH}$ removes the unoxidized silicon and causes the oxide structures to protrude even more with respect to the silicon base line [Fig. $3(\mathrm{c})]$. This is an indication that the formed motives are made of silicon dioxide. 

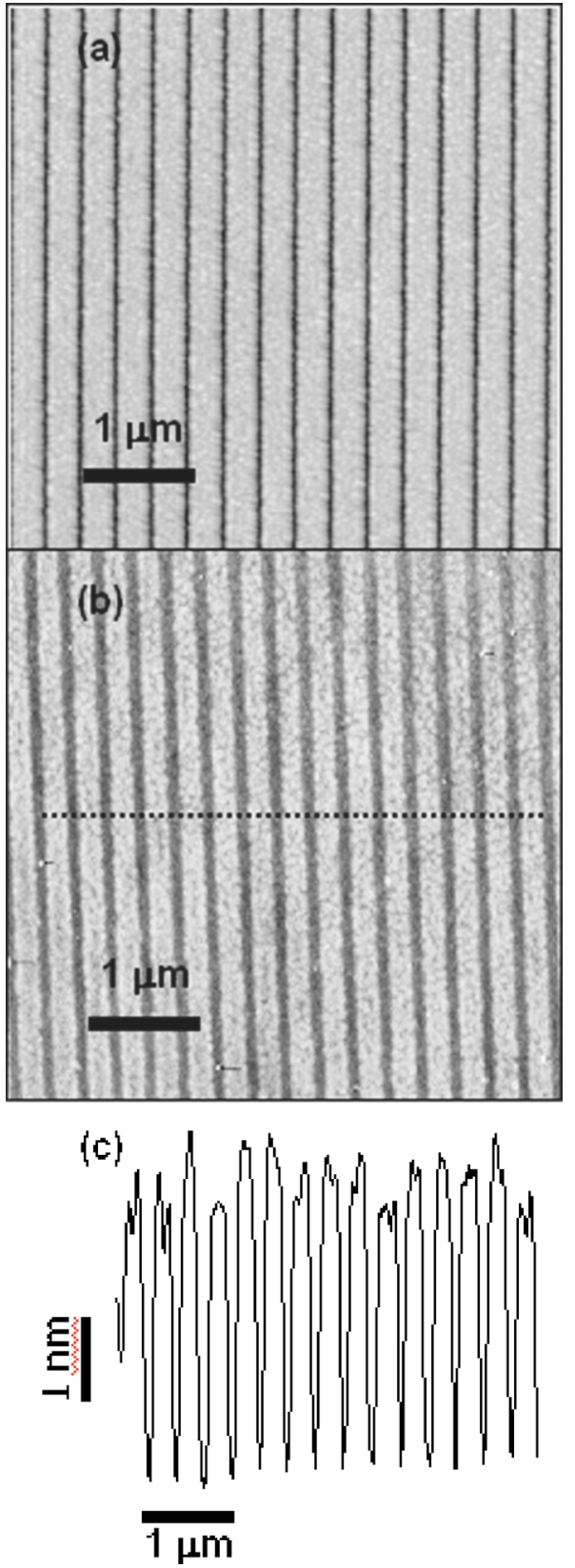

FIG. 4. (a) Amplitude modulation AFM image of gold coated silicon stamp. (b) AFM image of the replica. The local oxidation replica enhances the channels between the parallel lines. (c) Cross section along the line marked in (b).

Figure 4(a) is a zoom of one of the $20 \times 20 \mu \mathrm{m}^{2}$ stamp patches while Fig. 4(b) is the parallel oxidation replica. The cross section along the line marked in Fig. 4(b) shows that the oxide patterns are $4 \mathrm{~nm}$ in height [Fig. 4(c)].

In summary, we have developed an instrument for parallel oxidation nanolithography. The system allows us to pattern nanoscale silicon oxide motives over $1 \mathrm{~cm}^{2}$ regions. The parallel patterning process is rather fast because macroscopic regions are patterned in less than $1 \mathrm{~min}$. The instrument enables to control both the pressure exerted on the sample and the lateral positioning of the stamp on the sample. To demonstrate its performance, we have patterned arrays of parallel lines of several micrometers in length and a separation of $100 \mathrm{~nm}$. Atomic force microscopy images of the imprinting process reveal that the replicas reproduce faithfully the stamp periodic features.

This work was financially supported by the MCyT (Spain) (MAT2003-02655) and the EU integrated project NAIMO (Grant No. NMP4-CT-2004-500355).

${ }^{1}$ L. Pellegrino, I. Pallecchi, D. Marre, E. Bellingeri, and A. S. Siri, Appl. Phys. Lett. 81, 3849 (2002).

${ }^{2}$ N. Clement et al., Appl. Phys. Lett. 82, 1727 (2003).

${ }^{3}$ R. Crook, A. C. Graham, C. G. Smith, I. Farrer, H. E. Beere, and D. A. Ritchie, Nature (London) 424, 751 (2003).

${ }^{4}$ R. Garcia, M. Tello, J. F. Moulin, and F. Biscarini, Nano Lett. 4, 1115 (2004).

${ }^{5}$ C. F. Chen, S. D. Tzeng, H. Y. Chen, and S. Gwo, Opt. Lett. 30, 652 (2005).

${ }^{6}$ R. Garcia, R. V. Martinez, and J. Martinez, Chem. Soc. Rev. 35, 29 (2006).

${ }^{7}$ H. Kuramochi, K. Ando, T. Tokizaki, and H. Yokoyama, Appl. Phys. Lett. 84, 4005 (2004).

${ }^{8}$ M. Calleja, J. Anguita, R. Garcia, K. Birkelund, F. Perez-Murano, and J. A. Dagata, Nanotechnology 10, 34 (1999).

${ }^{9}$ G. Mori, M. Lazzarino, D. Ercolani, L. Sorba, S. Heuen, and A. Locatelli, J. Appl. Phys. 97, 114324 (2005).

${ }^{10}$ K. Matsumoto, Y. Gotoh, T. Maeda, J. A. Dagata, and J. S. Harris, Appl. Phys. Lett. 76, 239 (2000).

${ }^{11}$ S. Gwo, J. Phys. Chem. Solids 62, 1673 (2001).

${ }^{12}$ R. Maoz, E. Frydman, S. R. Cohen, and J. Sagiv, Adv. Mater. (Weinheim, Ger.) 12, 725 (2000).

${ }^{13}$ M. Tello, R. Garcia, J. A. Martín-Gago, N. F. Martinez, M. S. MartínGonzalez, L. Aballe, A. Baranov, and L. Gregoratti, Adv. Mater. (Weinheim, Ger.) 17, 1480 (2005).

${ }^{14}$ C. R. Kinser, M. J. Schmitz, and M. C. Hersam, Nano Lett. 5, 91 (2005).

${ }^{15}$ I. Suez, S. A. Backer, and J. M. J. Frechet, Nano Lett. 5, 321 (2005).

${ }^{16}$ T. Mühl, J. Kretz, I. Mönch, C. M. Schneider, H. Brückl, and G. Reiss, Appl. Phys. Lett. 76, 786 (2000).

${ }^{17}$ S. Hoeppener, R. Maoz, and J. Sagiv, Nano Lett. 3, 761 (2003).

${ }^{18}$ M. Cavallini, P. Mei, F. Biscarini, and R. Garcia, Appl. Phys. Lett. 83, 5286 (2003).

${ }^{19}$ A. Yokoo, J. Vac. Sci. Technol. B 21, 2966 (2003).

${ }^{20}$ N. Farkas, J. R. Comer, G. Zhang, E. A. Evans, R. D. Ramsier, S. Wight, and J. A. Dagata, Appl. Phys. Lett. 85, 5691 (2004). 